\title{
Imaging methods for the local lymphatic system of the axilla in early breast cancer in patients qualified for sentinel lymph node biopsy
}

\author{
Tomasz Nowikiewicz ${ }^{1}$, Andrzej Kurylcio ${ }^{2}$, Wojciech Polkowski², Wojciech Zegarski ${ }^{3}$ \\ ${ }^{1}$ Department of Clinical Breast Cancer and Reconstructive Surgery, The Franciszek Łukaszczyk Oncology Center, Bydgoszcz, Poland \\ ${ }^{2}$ Department of Surgical Oncology, Medical University of Lublin, Poland \\ ${ }^{3}$ Surgical Oncology Clinic, Collegium Medicum Nicolaus Copernicus University, The Franciszek Łukaszczyk Oncology Center, Bydgoszcz, Poland
}

\begin{abstract}
Breast cancer is the most common malignancy in women in well-developed countries. Despite a constant increase in its incidence, the percentage of patients diagnosed with the disease in the non-invasive stage is also rising. This allows more frequently for the use of breast-preserving surgical techniques, involving the breast and the regional lymphatic system.

According to current guidelines of expert panels and research societies, the recommended method of identifying the sentinel lymph node is the use of an isotope marker with a dye (a combined isotope and dye method). Cooperation with a nuclear medicine unit is essential (performing a preoperative lymphoscintigraphic scan to identify the lymphatic drainage basin and sentinel lymph node). In the case of smaller centers treating breast cancer, it can be associated with a number of difficulties, including organizational ones, and also increasing general treatment costs.

A possible solution to these problems is to use alternative techniques of visualizing the sentinel lymph node, which do not require a radiotracer. In this paper we discuss the currently available methods of mapping the lymphatic system of the axillary region in patients with early breast cancer. The review is limited to reporting on methods of proven (based on clinical research) high diagnostic value.
\end{abstract}

Key words: breast cancer, sentinel lymph node biopsy, imaging methods of lymphatic system.

\section{Introduction}

Breast cancer is the most common malignancy in women in Poland and other developed countries [1, 2]. The risk of occurrence is connected with older age and the peak incidence is observed in the post-menopausal period - the sixth to seventh decade of life [3]. In 2006, a total of 429,900 new cases of breast cancer were diagnosed in Europe, accounting for $28.9 \%$ of the overall number of cancer cases detected in that period [2]. At the same time, continuous growth in the incidence is observed; the trend is predicted to be maintained in future years [3, 4].

The long-term outcomes of breast cancer treatment depend on initial disease staging as one important prognostic factor [5].

In order to maintain the radical character of surgical treatment, diagnosing breast cancer requires the assessment of the status of lymph nodes within the axillary fossa at the side of the primary tumor lesion. The breast and axilla ultrasound examination is the most frequently performed radiological test, repeatable and easily accessible, to check regional lymph node status [6].
As demonstrated by the results of the NSABP B-32 study, the therapeutic management of choice in patients at the initial stage of the disease, i.e. without confirmed metastases to axillary lymph nodes (cNO), is the sentinel lymph node biopsy (SLNB) [7]. Compared to the resection of the entire lymphatic system within the axillary fossa, it is associated with a significantly lower rate of complications from the upper limb on the side of surgery, and thus with a better quality of life of patients after surgery. Similar conclusions were also presented by other authors [8-10].

\section{Alternative methods of imaging of the axillary lymphatic system in patients with early-stage breast cancer}

In line with current guidelines of research teams and associations, the recommended method for identification of the sentinel node is the combined use of a radioisotope solution (technetium ${ }^{99 \mathrm{~m}} \mathrm{Tc}$ ) and an aniline dye (isosulfan) or methylene blue solution - the combined radioisotope/dye method [11-13]. This requires col- 
laboration with Departments of Nuclear Medicine (for the purpose of performing a preoperative lymphoscintigraphic scan visualizing lymphatic flow and location of the sentinel lymph node), which, in the case of smaller sites providing breast cancer treatment, may be associated with a number of difficulties, including organizational difficulties (extended hospitalization times, the need to transport patients between medical centers, etc., and thus to increased treatment costs).

A problem associated with the isotopic method that is rarely taken into consideration is possible temporary inaccessibility of the radiomarker. The limited number of its production sites ${ }^{99 \mathrm{~m} T c}$ is manufactured at 5 sites: in Belgium, Canada, France, the Netherlands and South Africa) is the cause of transient deficiencies in marker supply [14]. Such deficiencies are due to technical reasons (e.g. service interruptions in operations of nuclear reactors) as well as to logistic problems with the transport of the material. The rising demand for the radiomarker is also an important factor here. In the US alone, nearly $80 \%$ of about 20 million nuclear medicine procedures carried out each year require the use of technetium isotope [14]. Further advances in the applicability of the sentinel lymph node biopsy in the treatment of other types of malignancies may significantly increase this demand.

The solution to these problems may consist in the development of alternative techniques for visualization of lymph nodes that would not require the use of radiomarkers [15]. The ability to administer the marker in the operating room immediately before the procedure allows one to avoid all the inconveniences characteristic for the use of radioisotopes. Besides those listed above, there is also the problem of radiation exposure of patients and medical personnel. The use of radiomarkers is also subject to considerable regulatory control (the requirement for specialist training of medical staff, supervision of medical waste disposal, including post-operative waste disposal). Moreover, the radioisotope method does not guarantee appropriate quality of preoperative imaging [15].

A promising, non-radioactive method for visualization of lymphatic flow into the axillary nodes that thus allows for independence from nuclear medicine departments is the use of a novel marker, Sienna+, which contains superparamagnetic iron oxide (SPIO), together with a handheld magnetometer (SentiMag). Sienna+ and SentiMag are patented by Endomagnetics, Ltd. and were granted the CE marking as class Ila medical devices.

Iron oxide is a non-toxic component of the contrast agent which has been in use in magnetic resonance imaging for many years $[16,17]$. The molecules of iron oxide are coated with carboxydextran to prevent accumulation of the marker and maintain its biocompatibility. The diameter of Sienna+ molecules is $60 \mathrm{~nm}$, allowing for their fast transport along the lymphatic system.
Surgical procedures using the mentioned iron oxide are preceded by subcutaneous or interstitial injection of Sienna+ (subzonal/perizonal). The injection is accompanied by a 5 -minute massage of the administration site. After another 15-20 minutes, a magnetometer (SentiMag) may be used for identification of marker deposits formed in the lymph nodes within the axillary fossa. Due to the small size of the molecules, Sienna+ is characterized by its capacity for fast transport within the lymphatic system, including equally fast filtration into the encountered lymph nodes. Therefore, sentinel node lymphadenectomy may be performed within one week after administration of the marker. The brownblack coloration of the tracer additionally facilitates visualization of the lymph nodes of interest during the procedure. As shown by the studies by Douek et al. [18] and Thill et al. [19], the use of Sienna+ marker and the SentiMag device during the SLNB procedure has diagnostic value that is comparable to the classic method. The results of both multicenter clinical studies unanimously demonstrated that the tested sentinel node identification methods lead to the same percentage of successful procedures (in the case of SPIO, the sentinel node detection rate was 98\% [19]).

The non-radioisotopic method for identification of the sentinel node is also possible by means of indocyanine green. Initially, the use of indocyanine green consisted in taking advantage of the dye's ability to deliver green staining of the lymph vessels and nodes (as an alternative to the aniline dye isosulfan). As demonstrated by Motomura et al. on the basis of an analysis of 172 breast cancer patients, the choice of this technique facilitated visualization of stained sentinel nodes in $74 \%$ of cases (method sensitivity of 95\%) [20]. In addition, as revealed by observations made by the same team, the use of indocyanine green together with a radioisotope (technetium ${ }^{99 \mathrm{~m} T c}$ ) facilitated visualization of sentinel nodes in $95 \%$ of patients [21].

Currently, use is made of another property of indocyanine green, namely its fluorescence upon excitation by infrared radiation (wavelengths of ca $800-840 \mathrm{~nm}$ ). Surgical procedures making use of indocyanine green require a light-emitting diode (LED) and a charge-coupled device (CCD) for detection of the excited radiation. Intravenous administration of the marker facilitates intraoperative assessment of tissue vascularity used in cosmetic and reconstruction surgery [22], cardiac surgery [23] and transplantology [24].

Due to the small molecule size $(1.2 \mathrm{~nm})$ and solubility in water, indocyanine green is easily drained into the lymphatic system [25]. Deposition of indocyanine green in the vicinity of the tumor - extravascular, i.e. subcutaneous injection) should therefore facilitate mapping of lymphatic vessels leading to the sentinel node as well as visualization of the node itself. Studies conducted by Japanese researchers confirmed the correctness of 
the above assumptions. Administration of the marker in a group of 18 breast cancer patients and subsequent infrared fluorescence measurements facilitated identification of sentinel nodes in 17 cases (94\%) [26]. In one half of patients subjected to surgical treatment, the resected lymph node was stained green. The authors observed no false-negative results of the SLNB procedures as confirmed by axillary lymphadenectomies carried out in all patients.

As demonstrated by results of further analyses regarding the ability to visualize the lymphatic flow from the primary tumor region towards the axillary lymph nodes, the technique making use of indocyanine green is characterized by high clinical value, comparable to the classic method, in breast cancer patients [27, 28]. However, as suggested by authors of studies conducted to date, confirmation of an appropriate level of efficacy of the proposed procedure requires studies to be conducted in larger patient populations.

As shown in the studies conducted by Takamaru et al., an alternative way to visualize the sentinel node in a non-radioactive manner is the use of aniline dye Patent Blue V (isosulfan) or methylene blue solution alone [29]. In a group of 374 patients subjected to surgical treatment using this procedure, the treatment was completed by resection of the node of interest in $96.8 \%$ of cases. The sensitivity of the method was $96.4 \%$, with specificity of $100 \%$, and the rate of false-negative results was $3.6 \%$.

A much lower rate of identification of the sentinel node following the use of dye alone (isosulfan) was obtained by Cox et al. [30]. The method used for visualization allowed the node of interest to be identified in $80.3 \%$ of patients. Similar results were obtained by Kim et al., who presented a meta-analysis encompassing a total of 8059 breast cancer patients qualified for axillary lymph node conservation treatment [10]. In the case of the dye method, the rate of identification of the sentinel node was $83.1 \%$ (vs. $91.9 \%$ for the combined radioisotope/dye method). At the same time, $10.9 \%$ of false negative results of biopsy procedures were observed (vs. 7.0\% for the combined radioisotope/dye method).

Besides the lower efficacy in sentinel node identification as compared to the reference method, the dye method is burdened by other disadvantages as well. Administration of the dye may lead to hypersensitivity reactions of varied intensity (this pertains mainly to isosulfan) [31-33]. The incidence of hypersensitivity reactions is 1.8 to $2.5 \%$ of cases [31-34]. As demonstrated by the studies conducted by Cimmino et al., symptoms of anaphylactic shock may develop in nearly one half of the patients [33]. Negative consequences of the use of the dye also include a possibility of developing a permanent tattoo at the injection site. This is the case particularly for intradermal administrations when the administration site is not fully resected during the procedure [31-34].
The use of methylene blue instead of isosulfan during the SLNB procedure is several times less expensive [14]. It is also associated with less frequent and less intense hypersensitivity reactions [33]. However, due to the possibility of causing skin damage (ulceration or necrotic lesions of the skin) at the administration site, this marker has not found wider use as compared to the aniline dye [35]. As observed in the studies conducted by Bleicher et al., similar changes may also occur in case of interstitial peritumoral injection of methylene blue [36]. They manifested as clearly defined skin erythema and telangiectasias in $11.8 \%$ of cases in the group of 78 patients subjected to the SLNB procedure. The described adverse effects are due to marker-induced vasospasms (methylene blue was shown to be a nitric oxide inhibitor) and, as a consequence, are directly related to ischemic changes occurring in tissues in the administration area.

Due to the aforementioned problems, applicability of the dye-based method as the only method for sentinel node detection was significantly limited $[14,37]$.

\section{Conclusions}

When introducing sentinel lymph node biopsy for routine use, it is recommended to use a method allowing for appropriately high rates of identification of the lymph nodes of interest (i.e. above 90\%). Also important is the requirement of a low percentage of false negative results of SLNB procedures, not higher than $5 \%$ [38]. According to current guidelines, the procedure requires the use of a combined identification method (i.e. the radioisotope/dye method).

The good quality of the preoperative lymphatic mapping is the factor determining the proper identification of the sentinel lymph node especially in patients who underwent previous breast surgery (assumed nonanatomical or unsettled axillary lymph spread) [39].

Continuation of studies mentioned in this review will clarify whether the currently recommended method for visualization of sentinel nodes could be replaced by a safe yet efficient alternative procedure.

\section{Disclosure}

Authors report no conflict of interest.

\section{References}

1. Didkowska J, Wojciechowska U. Breast cancer in Poland and Europe population and statistics. Nowotwory J Oncol 2013; 63: 111-118.

2. Ferlay J, Autier P, Boniol M, et al. Estimates of the cancer incidence and mortality in Europe in 2006. Ann Oncol 2007; 18: 581-592.

3. Leśniczak B, Krasomski G, Oszukowski P, et al. Incidence of and mortality from breast cancer among women in Poland in the years 2001-2010. Prz Menopauzalny 2014; 13: 344-347.

4. Maciejczyk A, Szelachowska J, Ekiert M, et al. Analysis of BCRP expression in breast cancer patients. Ginekol Pol 2012; 83: 681-687. 
5. Nowikiewicz T, Wiśniewska M, Wiśniewski M, et al. Overall survival and disease-free survival in breast cancer patients treated at Oncology Centre in Bydgoszcz - analysis of an over six-year long follow-up. Contemp Oncol (Pozn) 2015; 19: 284-289.

6. Nowikiewicz T, Nowak A, Wiśniewska M, et al. Diagnostic value of preoperative axillary lymph node ultrasound assessment in patients with breast cancer qualified for sentinel lymph node biopsy. Wideochir Inne Tech Maloinwazyjne 2015; 10: 170-177.

7. Krag DN, Anderson SJ, Julian TB, et al. Sentinel-lymph-node resection compared with conventional axillary-lymph-node dissection in clinically node-negative patients with breast cancer: overall survival findings from the NSABP B-32 randomised phase 3 trial. Lancet Oncol 2010 11: 927-933.

8. Taradaj J, Halski T, Zduńczyk M, et al. Evaluation of the effectiveness of kinesio taping application in a patient with secondary lymphedema in breast cancer: a case report. Prz Menopauzalny 2014; 13: 73-77.

9. Malicka I, Rosseger A, Hanuszkiewicz J, Woźniewski M. Kinesiology Taping reduces lymphedema of the upper extremity in women after breast cancer treatment: a pilot study. Prz Menopauzalny 2014; 13: 221-226.

10. Kim T, Giuliano AE, Lyman GH. Lymphatic mapping and sentinel lymph node biopsy in early-stage breast carcinoma: a metaanalysis. Cancer 2006; 106: 4-16.

11. Goldhirsch A, Wood WC, Coates AS, et al. Strategies for subtypesdealing with the diversity of breast cancer: highlights of the St Gallen International Expert Consensus on the Primary Therapy of Early Breast Cancer 2011. Ann Oncol 2011; 22: 1736-1747.

12. Jassem J, Krzakowski M. Rak piersi. In: Zalecenia postępowania diagnostyczno-terapeutycznego w nowotworach złośliwych 2013 rok. Krzakowski M, Warzocha K (eds.). Onkologia w praktyce klinicznej. Tom 1. Via Medica, Gdańsk 2013; 211-263.

13. Herman K, Marczyk E. Rak piersi - rekomendacje Konsultanta Krajowego w dziedzinie chirurgii onkologicznej. In: Rak piersi - postępy diagnostyki i leczenia. Zegarski W, Jastrzębski T, Nowikiewicz T (eds.) Medipage, Warszawa 2013; 1-6.

14. Kelley LM, Holmes DR. Tracer agents for the detection of sentinel lymph nodes in breast cancer: Current concerns and directions for the future. J Surg Oncol 2011; 104: 91-96.

15. Thill M, Baumann K. New technologies in breast cancer surgery. Breast Care 2012; 7: 370-376.

16. Gunasekera UA, Pankhurst QA, Douek M. Imaging applications of nanotechnology in cancer. Target Oncol 2009; 4: 169-181.

17. Johnson L, Gunasekera A, Douek M. Applications of nanotechnology in cancer. Discov Med 2009; 9: 374-379.

18. Douek M, Klaase J, Monypenny I, et al. Sentinel node biopsy using a magnetic tracer versus standard technique: The SentiMAG Multicentre Trial. Ann Surg Oncol 2014; 21: 1237-1245.

19. Thill M, Kurylcio A, Welter R, et al. The Central-European SentiMag study: sentinel lymph node biopsy with superparamagnetic iron oxide (SPIO) vs. radioisotope. Breast 2014; 23: 175-179.

20. Motomura K, Inaji H, Komoike Y, et al. Sentinel node biopsy guided by indocyanine green dye in breast cancer patients. Jpn J Clin Oncol 1999; 29: 604-607.

21. Motomura K, Inaji H, Komoike Y, et al. Combination technique is superior to dye alone in identification of the sentinel node in breast cancer patients. J Surg Oncol 2001; 76: 95-99.

22. Lee BT, Matsui A, Hutteman M, et al. Intraoperative nearinfrared fluorescence imaging in perforator flap reconstruction: Current research and early clinical experience. J Reconstr Microsurg 2010; 26: 59-65.

23. Reuthebuch O, Häussler A, Genoni M, et al. Novadaq SPY: Intraoperative quality assessment in off-pump coronary bypass grafting. Chest 2004; 125: 418-424.

24. Sekijima M, Tojimbara T, Sato S, et al. An intraoperative fluorescent imaging system in organ transplantation. Transplant Proc 2004; 36: 2188-2190.

25. Gurfinkel M, Thompson AB, Ralston W, et al. Pharmacokinetics of ICG and $\mathrm{HPPH}$-car for the detection of normal and tumor tissue using fluorecence, near-infrared reflectance imaging: a case study. Photochem Photobiol 2000; 72: 94-102.

26. Kitai T, Inomoto T, Miwa M, Shikayama T. Fluorescence navigation with indocyanine green for detecting sentinel lymph nodes in breast cancer. Breast Cancer 2005; 12: 211-215.
27. Murawa D, Hirche C, Dresel S, Hünerbein M. Sentinel lymph node biopsy in breast cancer guided by indocyanine green fluorescence. $\mathrm{Br} J \mathrm{Surg}$ 2009; 96: 1289-1294.

28. Tagaya N, Yamazaki R, Nakagawa A, et al. Intraoperative identification of sentinel lymph nodes by near-infrared fluorescence imaging in patients with breast cancer. Am J Surg 2008; 195: 850-853.

29. Takamaru T, Kutomi G, Satomi F, et al. Use of the dye-guided sentinel lymph node biopsy method alone for breast cancer metastasis to avoid unnecessary axillary lymph node dissection. Exp Ther Med 2014; 7: 456-460.

30. Cox CE, Bass SS, McCann CR, et al. Lymphatic mapping and sentinel lymph node biopsy in patients with breast cancer. Annu Rev Med 2000; 51: 525-542.

31. Albo D, Wayne JD, Hunt KK, et al. Anaphylactic reactions to isosulfan blue dye during sentinel lymph node biopsy for breast cancer. Am J Surg 2001; 182: 393-398.

32. Raut CP, Daley MD, Hunt KK, et al. Anaphylactoid reactions to isosulfan blue dye during breast cancer lymphatic mapping in patients given preoperative prophylaxis. J Clin Oncol 2004; 22: 567-568.

33. Cimmino VM, Brown AC, Szocik JF, et al. Allergic reactions to isosulfan blue during sentinel node biopsy - a common event. Surgery 2001; 130 : 439-342.

34. Raut CP, Hunt KK, Akins JS, et al. Incidence of anaphylactoid reactions to isosulfan blue dye during breast carcinoma lymphatic mapping in patients treated with preoperative prophylaxis: results of a surgical prospective clinical practice protocol. Cancer 2005; 104: 692-699.

35. Stradling B, Aranha G, Gabram S. Adverse skin lesions after methylene blue injections for sentinel lymph node localization. Am J Surg 2002; 184: 350-352.

36. Bleicher RJ, Kloth DD, Robinson D, Axelrod P. Inflammatory cutaneous adverse effects of methylene blue dye injection for lymphatic mapping/ sentinel lymphadenectomy. J Surg Oncol 2009; 99: 356-360.

37. Nowikiewicz T, Biedka M, Krajewski E, et al. Analysis of selected problems of sentinel lymph node biopsy in patients with breast cancer. Curr Gynecol Oncol 2012; 10: 296-306.

38. Schwartz GF, Giuliano AE, Veronesi $U$ and the Consensus Conference Committee. Proceedings of the consensus conference on the role of sentinel lymph node biopsy in carcinoma of the breast, April 19-22, 2001, Philadelphia, Pennsylvania. Cancer 2002; 94: 2542-2551.

39. Qin R, Zhang Q, Weng J, et al. Treatment and prognosis for retrograde cervical lymph node metastases in breast cancer. Contemp Oncol (Pozn) 2015; 19: 154-156. 